\title{
FRATTINI SUBALGEBRAS OF FINITELY GENERATED SOLUBLE LIE ALGEBRAS \\ BY
}

\author{
RALPH K. AMAYO
}

\begin{abstract}
This paper is motivated by a recent one of Stewart and Towers [8] investigating Lie algebras with "good Frattini structure" (definition below). One consequence of our investigations is to prove that any finitely generated metanilpotent Lie algebra has good Frattini structure, thereby answering a question of Stewart and Towers and providing a complete Lie theoretic analogue of the corresponding group theoretic result of Phillip Hall. It will also be shown that in prime characteristic, finitely generated nilpotent-by-finite-dimensional Lie algebras have good Frattini structure.
\end{abstract}

1. Preliminaries. We employ the notation of Amayo and Stewart [3]. For a fixed ground field $\mathfrak{t}, \mathfrak{A}, \mathfrak{F}, \mathbb{S}, \mathfrak{R}$ denote the classes of abelian, finite-dimensional, finitely generated, and nilpotent Lie, algebras respectively. If $\mathfrak{X}$ and $\mathfrak{Y}$ are classes of Lie algebras, then $\mathfrak{X Y}$ is the class of all Lie algebras $L$ having an ideal $I \in \mathfrak{X}$ with $L / I \in \mathfrak{Y}$. We write $\mathfrak{X}^{2}$ for the class $\mathfrak{X X}$, and, in general, $\mathfrak{X}^{n+1}=\mathfrak{X}^{n} \mathfrak{X}$. We also refer to $\mathfrak{X} \mathfrak{Y}$ as the class of $\mathfrak{X}$-by- $\mathfrak{Y}$ Lie algebras, and $\mathfrak{X}^{2}$ is the class of meta- $\mathfrak{X}$ algebras. Thus $\mathfrak{R}^{2}$ is the class of metanilpotent Lie algebras.

The symbol $L$ will denote a Lie algebra of arbitrary dimension defined over the field $t$. The notation $A \subseteq L, A<L, A \triangleleft L, A$ si $L$ means that $A$ is a subset, subalgebra, ideal, and subideal of $L$, respectively. By $A<\cdot L$ we mean that $A$ is a maximal subalgebra of $L$. If $A, B \subseteq L$, then $[A, B]$ is the subspace of $L$ spanned by all $[a, b]$ with $a \in A$ and $b \in B,\left[A,_{n+1} B\right]=\left[\left[A,{ }_{n} B\right], B\right]$ and $\left[A,,_{0} B\right]=A ;\left[a, a_{0} b\right]=a$ and $\left[a,_{n+1} b\right]=\left[\left[a,{ }_{n} b\right], b\right]$.

The Frattini subalgebra $F(L)$ is the intersection of the maximal subalgebras of $L$ or is $L$ if there are no maximal subalgebras. The Frattini ideal $\Phi(L)$ is the largest ideal of $L$ contained in $F(L)$. In general, $F(L) \neq$ $\Phi(L)$.

A chief factor of $L$ is a pair $(H, K)$ of ideals of $L$ such that $H>K$ and no ideal of $L$ lies properly between $H$ and $K$. We also refer to the corresponding factor ideal $H / K$ of $L / K$ as the chief factor.

If $A \triangleleft B<L$, then

Received by the editors November $12,1976$.

AMS (MOS) subject classifications (1970). Primary 17B30, 17B65, $17 \mathrm{~B} 05$.

Key words and phrases. Lie algebra, Frattini subalgebra.

- American Mathematical Society 1978 


$$
C_{L}(B / A)=\{x \in L:[B, x] \subseteq A\} .
$$

If $A$ and $B$ are ideals of $L$, then $C_{L}(B / A)$ is also an ideal of $L$.

$$
\psi(L)=\cap\left\{C_{L}(H / K): H / K \text { is a chief factor of } L\right\} .
$$

The Hirsch-Plotkin radical $\rho(L)$ is the unique maximal locally nilpotent ideal of $L$. The Fitting radical $\nu(L)$ is the sum of the nilpotent ideals of $L$. We always have $\nu(L)<\rho(L)$. We set

$$
\tilde{\nu}(L) / \Phi(L)=\nu(L / \Phi(L)) \text { and } \tilde{\psi}(L) / \Phi(L)=\psi(L / \Phi(L)) .
$$

We say that $L$ has good Frattini structure if $\nu(L)$ is nilpotent and $\nu(L)=$ $\rho(L)=\psi(L)=\tilde{\nu}(L)$.

Let $U=U(L)$ be the universal enveloping algebra of $L$ and let $A$ be an $L$-module (and hence $U$-module). For a two-sided ideal $I$ of $U$ we let

$$
Z(L: I)=Z^{*}(L ; I) / I
$$

be the center of $U / I$.

We say that $B / C$ is a chief factor submodule of $A$ in case $B$ and $C$ are submodules of $A, B>C$ and no submodule lies strictly between $B$ and $C$. If $N \subseteq M$ are submodules of $A$, then

$$
\operatorname{Ann}_{U}(M / N)=\{u \in U: M u \subseteq N\} .
$$

We define

$$
\psi(A ; U)=\cap\left\{\operatorname{Ann}_{U}(B / C): B / C \text { is a chief factor submodule }\right\} .
$$

Clearly $\psi(A ; U)$ is a two-sided ideal of $U(L)$, and if we consider $L$ as a module over itself under the adjoint action, then

$$
L \cap \psi(L ; U)=\psi(L) \text {. }
$$

We shall prove

TheOREM A. If $L \in \mathfrak{F}$ and $A$ is a finitely generated $L$-module, then there is an integer $n$ such that $A\left(\psi(A ; U) \cap Z^{*}\left(L ; \operatorname{Ann}_{U}(A)\right)\right)^{n}=0$.

Combining this with the fact that the universal enveloping algebras of nilpotent Lie algebras have centralizing sets of generators will yield

Theorem B. If $L \in \mathfrak{F} \cap \mathfrak{R}$ and $A$ is a finitely generated Lie algebra, then there is an integer $n$ such that $A(\psi(A ; U))^{n}=0$.

When $\mathrm{f}$ has prime characteristic, we can prove more, namely:

THEOREM C. If $L \in \mathfrak{F}$ over a field of prime characteristic and if $A$ is a finitely generated $L$-module, then there is an integer $n$ such that $A(\psi(A ; U))^{n}=0$.

Applications of these results yield: 
ThEOREM D. Any finitely generated metanilpotent Lie algebra has good Frattini structure.

ThEOREM E. Any finitely generated nilpotent-by-finite-dimensional Lie algebra over a field of prime characteristic has good Frattini structure.

As is remarked in Stewart and Towers [8] we have

COROLlary F. The natural representation of $L$ on $\nu(L)$ induces a faithful representation of $L / \nu(L)$ on $\nu(L) / \Phi(L)$ whenever $L \in$ \&s $\cap \mathfrak{R}^{2}$.

2. The Frattini ideal. In Stewart and Towers [8] it is proposed (though their proof is incorrect) that if $L \in \mathbb{S} \cap \mathfrak{N}^{2}$, then $\nu(L)^{2} \subseteq \Phi(L)$. This result is, in fact, true for any Lie algebra as we now show.

Proposition 2.1. Let $L$ be any Lie algebra. Then:

(a) $\nu(L)<\psi(L)$.

(b) $[\nu(L), \psi(L)] \subseteq \Phi(L)$.

(c) $[\tilde{v}(L), \tilde{\psi}(L)] \subseteq \Phi(L)$.

(d) If $\tilde{\psi}(L) / \Phi(L)$ is a sum of solvable ideals of $L / \Phi(L)$, then $\tilde{\nu}(L)=\tilde{\psi}(L)$.

In particular, $\tilde{\nu}(L) / \Phi(L)$ is abelian and $\nu(L)^{2} \subseteq \Phi(L)$.

Proof. (a) Suppose that $I$ is a nilpotent ideal and $H / K$ a chief factor of $L$. Then $[H, I]+K$ is an ideal of $L$ between $H$ and $K$. If $H=[H, I]+K$, then $H=\left[H,,_{2} I\right]+K=\cdots=\left[H,{ }_{n} I\right]+K \subseteq I^{n}+K$ for any positive integer $n$. Since $I^{n}=0$ for some $n$ this would imply that $H=K$, a contradiction. Thus $[H, I]+K=K$ and $[H, I] \subseteq K$ and $I \subseteq \psi(L)$.

(b) Suppose that $M<\cdot L$ and $[\nu(L), \psi(L)] \nsubseteq M$. Then there is a nilpotent ideal $I$ such that $[I, \psi(L)] \nsubseteq M$. If $I^{2} \nsubseteq M$, then $L=I^{2}+M$, whence $I=I^{2}+I \cap M=I^{r}+I \cap M$ for all $r$ and so $I=I \cap M$, a contradiction. Thus $I^{2} \subseteq M$ and $I^{2} \subseteq I \cap M \neq I, L=I+M$ and $I \cap M$ is an ideal of $L$. As $M<\cdot L, I / I \cap M$ is a chief factor of $L$ and so $[I, \psi(L)] \subseteq I$ $\cap M \subseteq M$, a contradiction. So $[\nu(L), \psi(L)] \subseteq F(L)$, and since $[\nu(L), \psi(L)]$ is also an ideal of $L$, we have $[\nu(L), \psi(L)] \subseteq \Phi(L)$.

(c) follows from (b) and the definitions of $\tilde{\nu}(L)$ and $\tilde{\psi}(L)$.

(d) Suppose that $\tilde{\psi}(L) \neq \tilde{\nu}(L)$ and $\tilde{\psi}(L) / \Phi(L)$ is a sum of solvable ideals of $L / \Phi(L)$. Then there is an ideal $I$ of $L$ contained in $\tilde{\psi}(L)$ such that the derived length of $I / \Phi(L)$ is minimal with respect to $I^{2} \nsubseteq \Phi(L)$. Then there is $M<\cdot L$ such that $L=I^{2}+M$. Now $\left(\left(I^{2}\right)+\Phi(L)\right) \neq I$ (else as $\Phi(L) \triangleleft L$ we would have $I=I^{(r)}+\Phi(L)$ for all $r$ and so $\left.I=\Phi(L)\right)$, and hence $\left(I^{2}+\Phi(L)\right)^{2} \subseteq$ $\Phi(L)$. In particular, if $J=I^{2}+\Phi(L)$, then $J \cap M \triangleleft J$ and so $J \triangleleft L$. Now $J / J \cap M$ is isomorphic to the chief factor $J / \Phi(L) /(J \cap M / \Phi(L))$ of $L / \Phi(L)$ and, hence, $[J, \tilde{\psi}(L)] \subseteq J \cap M$. This implies that $(\tilde{\psi}(L))^{2} \subseteq M$, since $\tilde{\psi}(L)=J+\tilde{\psi}(L) \cap M$ and $J^{2} \subseteq M$. Thus $L=J+M \subseteq(\tilde{\psi}(L))^{2}+$ 
$M \subseteq M$, a contradiction. This proves (d). The rest follows from (a)-(d).

LemmA 2.2. If $L \in \mathfrak{F}$ and $A$ is an irreducible L-module then $Z\left(L ; A \operatorname{Ann}_{U}(A)\right)$ is finite dimensional over $\mathrm{Z}$.

Proof. If $\mathfrak{t}$ has prime characteristic, then, by a result of Curtis $[5$, p. 952], $A$ and, hence, $U / \operatorname{Ann}_{U}(A)$ is finite dimensional over $\mathrm{f}$.

If $\mathfrak{t}$ has characteristic zero, then by Proposition 4.1.7 of Dixmier [7, p. 131], $Z\left(L ; \operatorname{Ann}_{U}(A)\right)$ is finite dimensional.

TheOREM 2.3. Let $L \in \mathfrak{F}$ and $A$ be a finitely generated $L$-module. Suppose that $\theta$ is an $L$-module endomorphism of $A$ such that $B \theta \subseteq C$ for any chief factor submodule $B / C$ of $A$. Then there is an $n$ such that $A \theta^{n}=0$.

Proof. Let $X=L \oplus \mathfrak{f}$ so that $A$ is a finitely generated $X$-module. Set $V=U(X)=U(L) \otimes_{\ell} \mathfrak{f}[\theta]$. Then if $I=\operatorname{Ann}_{V}(A), \theta+I \in Z(X ; I)$. Evidently every $L$-submodule of $A$ is an $X$-submodule. Suppose it is false that $A \theta^{n}=0$ for some $n$. Now $X$ is finite dimensional and so $A$ is a noetherian $X$-module, whence there is a submodule $N$ of $A$ maximal with respect to $A \theta^{n} \nsubseteq N$ for any $n$.

By replacing $A$ by $A / N$ we may assume that if $0 \neq B \subseteq \subseteq_{X} A$ then $A \theta^{n} \subseteq B$ for some n. Thus if $a \in A$ and $a \theta=0$, then $a V \theta=a \theta V=0$ and so $a=0$. Thus $\theta$ is a $V$-module monomorphism of $A$.

By the proof of Theorem 3.3 of Stewart and Towers [8, p. 214] we can embed $A$ in an $X$-module $M$ such that $M=A \mathfrak{t}[T]=A U(X \oplus \mathbb{t} T)$, is a finitely generated $(X \oplus \mathfrak{t})$-module, $\theta$ is an $(X \oplus \mathfrak{t})$-module automorphism of $M$ and $T \theta-1=\theta T-1 \in \operatorname{Ann}_{W}(M)$, where $W=U(X \oplus € T)$.

Let $N$ be a nonzero $W$-submodule of $M$. Suppose, if possible, that $N \cap A$ $=0$. Let $a_{0}, \ldots, a_{k} \in A$ and $b=a_{0}+a_{1} T+\cdots+a_{k} T^{k} \in N \backslash 0$ be such that $k$ is minimal. Then $k \neq 0$ and $a_{k} \notin A \theta$. But $b \theta^{k}=a_{0} \theta^{k}+a_{1} \theta^{k-1}$ $+\cdots+a_{1} \theta+a_{k} \in N \cap A=0$ and, hence, $a_{k} \in A \theta$, a contradiction. Thus $N \cap A \neq 0$, whence $A \theta^{n} \subseteq N \cap A$ for some $n$ and so $A=A \theta^{n} T^{n} \subseteq$ $N T^{n} \subseteq N$, so that $A \mathfrak{f}[T] \subseteq N$ and $N=M$. Thus $M$ is an irreducible $W$ module. By Lemma 2.2, $Z\left(M ; \operatorname{Ann}_{W}(M)\right)$ is finite dimensional, so there is a polynomial $f$ of minimal degree with $M f(\theta)=0$. Let $f(t)=\lambda_{0} t^{n}+\cdots+$ $\lambda_{n}$. As $\theta$ is an automorphism of $M$ we have $\lambda_{n} \neq 0$. If $a \in A$, then

$$
a=\lambda_{n}^{-1}\left(\lambda_{n} a\right)=-\lambda_{n}^{-1}\left(\left(f(\theta)-\lambda_{n}\right) a\right) \in A \theta \text {. }
$$

So $A=A \theta$ and, therefore, $A$ is an irreducible $X$-module, whence $A$ is an irreducible $L$-module and so $A=A \theta=0$, a contradiction. This proves the result.

The proof of Theorem 2.3 also yields the following useful corollary:

Corollary 2.4. Let $L \in \mathfrak{F}, A$ a finitely generated $L$-module and $z+I \in$ 
$Z\left(L ; \operatorname{Ann}_{U}(A)\right)$. If $B$ is any submodule of $A$ maximal with respect to $A z^{n} \nsubseteq B$ for any $n$, then $B$ is a maximal submodule of $A$.

Let $U$ be an arbitrary associative $t$-algebra and $A$ a $U$-module. Then we may define $\psi(A ; U)$ as before and $Z$ as the center of $U$. Then $U$ is said to have the chief annihilator property if $z \in \psi(A ; U) \cap Z$ and $A$ a finitely generated $U$-module implies $A z^{n}=0$ for some $n$.

We denote by CAP the class of Lie algebras $L$ such that $U(L)$ has the chief annihilator property. Evidently Theorem 2.3 states a stronger property, namely that if $z \in \psi(A ; U) \cap Z^{*}(L ; \operatorname{Ann}(U))$ then also $A z^{n}=0$ for some $n$. We refer to the class of Lie algebras with this property as SCAP (strong chief annihilator property). Then we have

Corollary 2.5. $\mathfrak{F}<$ SCAP.

We leave open the question of whether or not the inclusion is strict. We note also that SCAP is closed under homomorphic images.

\section{Proofs of the main results.}

Proof of Theorem $A$. Let $L \in \mathfrak{F}$ and $A$ be a finitely generated $L$-module and set $N=\psi(A ; U) \cap Z^{*}\left(L ; \operatorname{Ann}_{U}(A)\right)$. Clearly each element of $Z^{*}(L$; $\left.\operatorname{Ann}_{U}(A)\right)$ induces an $L$-module endomorphism of $A$ and so if $z \in N$, then by Theorem 2.3 there is an $n=n(z)$ such that $A z^{n}=0$. Using standard arguments and the fact that $N \bmod \operatorname{Ann}_{U}(A)$ is commutative, it follows that given any finite-dimensional subspace $S$ of $N$ there exists $n=n(S)$ such that $A S^{n}=0$. Now $U(L)$ has the maximal condition on right ideals and so $U N=N U=S U=U S\left(\bmod \operatorname{Ann}_{U}(A)\right)$ for some finite-dimensional subspace $S$ of $U$, whence $A(N U)^{n}=A(S U)^{n} \subseteq A S^{n} U=0$ for some $n$ and Theorem $A$ is proved.

If $L$ is a Lie algebra and $I$ is an ideal of $U(L)$, then a subspace $S$ of $U(L)$ is said to be $L$-invariant $(\bmod I)$ if $[s, x]=s x-x s \in S+I$ for any $x \in L$ and $s \in S$. Now if $L$ is nilpotent then for any $u$ in $U$ there is a $c$ minimal with respect to $[u, c L]=0$. From this it follows easily that

$$
\begin{aligned}
& \text { if } L \text { is nilpotent and } S \text { is } L \text {-invariant } \bmod I \text {, then either } \\
& S \subseteq I \text { or else }(S+I) \cap Z^{*}(L ; I) \nsubseteq I \text {. }
\end{aligned}
$$

Proof of Theorem B. Let $L \in \mathfrak{F} \cap \mathfrak{N}, U=U(L)$, and $A$ be a finitely generated submodule and $M=\psi(A ; U)$. Clearly if $E$ is a submodule or quotient module of $A$, then $M \subseteq \psi(E ; U)$. Suppose that $A M^{n} \neq 0$ for any $n$. As $A$ is a noetherian $U$-module we may replace $A$ by a suitable quotient of $A$ and assume then without loss of generality that if $B$ is a nonzero submodule of $A$ then $A M^{n} \subseteq B$ for some $n$ depending on $B$. Consider the set of ideals of $U$ which are annihilators of nonzero submodules of $A$ and let $I$ be a maximal 
element ( $U$ is noetherian) and $B$ a nonzero submodule of $A$ with $I=$ $\operatorname{Ann}_{U}(B)$. If $M \subseteq I$, then $A M^{n} \subseteq B$ and so $A M^{n+1}=0$ for some $n$, a contradiction. Assume that $M \nsubseteq I$. Then $M+I \neq I$ and $M+I$ is an $L$-invariant $(\bmod I)$ subspace of $\psi(B ; U)(M$ is an ideal of $U)$, and, hence, by (*), $N_{1}=(M+I) \cap Z^{*}(L ; I) \nsubseteq I$, and $N_{1} \subseteq N=\psi(B ; U) \cap Z^{*}(L ; I)$. By Theorem $A$ there exists $r$ minimal with respect to $B N^{r}=0$, whence $I<N U+I=U N+I \subseteq \operatorname{Ann}_{U}\left(B N^{r-1}\right)$ and $B N^{r-1}$ is a nonzero submodule of $A$, and this contradicts the maximality of $I$. This proves Theorem B.

Theorem $\mathrm{C}$ will follow from the more general Theorem 3.1 below. From Amayo and Stewart [3, pp. 225-232] we have the definition of the class Max-cu as consisting of all Lie algebras $L$ with the property that if $U=$ $U(L)$, then there exists a noetherian subring $R=\left\{\left[z_{1}, z_{2}, \ldots, z_{m}\right]\right.$ of the center of $U$ such that $U=R u_{1}+\cdots+R u_{n}$ is a finitely generated $R$ module. By the results of Curtis [5] and Amayo and Stewart [3, Chapter 11] we have the following facts:

(1) $\mathfrak{F} \cap \mathfrak{A}<$ Max-cu.

(2) In prime characteristic, $\&<$ Max-cu.

(3) For $L \in$ Max-cu, $U=U(L)$ and $R=\left\{\left[z_{1}, \ldots, z_{s}\right] \subseteq\right.$ center of $U$, such that $U=R u_{1}+\cdots+R u_{n}$;

(a) every irreducible $L$-module is finite dimensional;

(b) $U$ is a noetherian $R$-module and so satisfies the maximal conditions on left and right ideals;

(c) there exists $n=n(L)$ such that to any $u \in U$ there correspond $r_{1}, r_{2}, \ldots, r_{n} \in R$ for which

$$
u^{n}+r_{1} u^{n-1}+\cdots+r_{n-1} u+r_{n}=0 ;
$$

(d) every finitely generated $L$-module is noetherian.

Let $A$ be an $L$-module and $U=U(L)$. Clearly for any submodules $B, C$ of $A$ with $C \subseteq B$, we have $\psi(A ; U) \subseteq \psi(B / C ; U)$. We refer to the factor module $B / C$ and any $L$-module isomorphic to it (as $L$-modules) as an $L$-module section of $A$.

THEOREM 3.1. If $L \in$ Max-cu and $A$ is a finitely generated $L$-module, then there is an integer $m$ such that $A(\psi(A ; U))^{m}=0$.

Proof. Let $U=U(L)=R u_{1}+\cdots+R u_{n}$, where $R=\left\{\left[z_{1}, \ldots, z_{s}\right] \subseteq\right.$ center of $U$ as above and let $A$ be a finitely generated $L$-module for which the conclusion fails. Define

$\zeta=\{\psi(X ; U): X$ is an $L$-module section of $A$ and

$$
\left.X(\psi(X ; U))^{m} \neq 0 \text { for all } m\right\}
$$


As $U$ is noetherian there is a section $Y$ of $A$ for which $N=\psi(Y ; U)$ is a maximal element of $\zeta$. Pick a submodule $E$ of $Y$ maximal with respect to the property that $Y N^{m} \nsubseteq E$ for any $m$. Then if $A_{1}=Y / E$ we have $N \subseteq$ $\psi\left(A_{1} ; U\right)$ and so $A_{1} \psi\left(A_{1} ; U\right)^{m} \neq 0$ for any $m$, whence

$$
N=\psi\left(A_{1} ; U\right)=\psi(B ; U) \text { and } A_{1} N^{k} \subseteq B \text { for some } k,
$$

for any nonzero $L$-submodule $B$ of $A_{1}$. Let $\zeta_{1}=\left\{\operatorname{Ann}_{U}(B) ; 0 \neq B \subseteq_{L} A_{1}\right\}$ and pick $A_{2}$ such that $J=\operatorname{Ann}_{U}\left(A_{2}\right)$ is a maximal element of $\zeta_{1}$. Finally let

$$
\zeta_{2}=\left\{\operatorname{Ann}_{R}(a): 0 \neq a \in A_{2}\right\}
$$

and let $A_{3}=a U$ be such that $P=\operatorname{Ann}_{R}(a)$ is a maximal element of $\zeta_{2}$. By replacing $A$ by $A_{3}$ we now have:

(i) $N=\psi(A ; U)=\psi(B ; U)$ for any nonzero submodule $B$ of $A$;

(ii) if $0 \neq C \subseteq_{L} B \subseteq_{L} A$, then $B(\psi(A ; U))^{k} \subseteq C$ for some $k=k(C)$;

(iii) $J=\operatorname{Ann}_{U}(A)=\operatorname{Ann}_{U}(B)$ if $0 \neq B \subseteq_{L} A$;

(iv) $P=\operatorname{Ann}_{R}(A)=\operatorname{Ann}_{R}(b)$ for all $0 \neq b \in A$.

Now let $u \in N \backslash 0$. Then there exist by $(* *), r_{1}, r_{2}, \ldots, r_{n} \in R$ such that

(v) $u^{n}+r_{1} u^{n-1}+\cdots+r_{n}=0$.

Suppose if possible that for some $i, r_{i} \notin P$ and let $i$ be maximal such and set $r=r_{i}$. Thus $r_{i+1}, \ldots, r_{n} \in P$ in case $i<n$. Then $r$ defines an $L$-module monomorphism of $A$ by (iv). As in the proof of Theorem 2.3 we can embed $A$ in an $(L \oplus \mathbb{f})$-module $M=A \mathfrak{f}[T]$ such that $r T=T r=$ identity map on $M$. By (v),

$$
\begin{array}{r}
r u^{n-i}+r_{i-1} u^{n-i+1}+\cdots+r_{1} u^{n-1}+u^{n} \\
=\text { element of } P U \subseteq J \subseteq \operatorname{Ann}(M),
\end{array}
$$

and hence

$$
M u^{n-i}=(M r) u^{n-i} \subseteq M u^{n-i+1} \subseteq M u^{n-i}
$$

Thus

$$
M u^{n-i}=\bigcap_{k=1}^{\infty} M u^{k}=M u^{n} .
$$

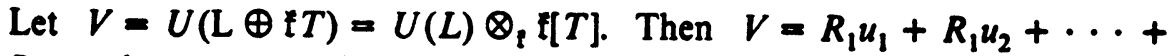
$R_{1} u_{n}$, where $R_{1}=R \otimes \mathfrak{f}[T] \simeq \mathfrak{\{}\left[z_{1}, \ldots, z_{s}, T\right] \subseteq$ center of $V$ and so $L_{1}=L$ $\oplus t T \in$ Max-cu. Suppose $B$ is a nonzero submodule of $M$. Then it is easy to check that $B \cap A \neq 0$, and so $A u^{k} \subseteq B \cap A$ for some $k(u \in N)$ by (ii) and hence $M u^{k} \subseteq B$. Thus if $\mu(M)=\bigcap\{B: 0 \neq B$ is a submodule of $M\}$ then $M u^{n} \subseteq \mu(M)$. Now if $\mu(M) \neq 0$, then $\mu(M)$ is an irreducible $L_{1}$-module and so is finite dimensional, whence $\mu(M) \cap A=D$ is a finite-dimensional nonzero submodule of $A$, so $D N^{l}=0$ for some $l$ and, hence, $A N^{k+l} \subseteq D N^{l}$ $=0$ for some $k$, a contradiction. So $M u^{n} \subseteq \mu(M)=0$, and $A u^{n}=0$. 
Thus given $u \in N \backslash 0$, we have that $u$ satisfies (v) for some $r_{j}$ in $R$. If all $r_{j}$ are in $P$, then $A u^{n}=0$. If not, then as above, $A u^{n}=0$ anyway. Therefore $N / J$ is a nil ideal of $U / J$ and $U / J$ satisfies the left and right ascending chain conditions and so, by a well-known result (see, for example, Divinsky [6, Theorem 16, p. 51]), $N / J$ is nilpotent, so $N^{m} \subseteq J$ for some $m$ or $A N^{m}=0$ for some $m$, a contradiction.

This proves Theorem 3.1.

\section{Applications.}

TheOREM D. Every finitely generated metanilpotent Lie algebra has good Frattini structure.

Proof. Let $L \in \mathbb{S} \cap \mathfrak{R}^{2}$. To show that $L$ has good Frattini structure it is enough, as in Stewart and Towers [8], to assume that $L \in \mathbb{S} \cap \mathfrak{A} \mathfrak{R}$ and to show that $\psi(L)=\nu(L)$. Let $A$ be an abelian ideal of $L$ for which $L / A \in$ (\$) $\cap \mathfrak{N}=\mathfrak{F} \cap \mathfrak{R}$, and let $N=\psi(L)$ and $X=N / A<Y+L / A$. Consider $A$ as an $L$-module under the adjoint action. Then $A$ is a finitely generated $L$-module (Amayo and Stewart [2]) and so a finitely generated $Y$-module. Now $X \subseteq \psi(A ; U)$, where $U=U(Y)$ and so, by Theorem $\mathrm{D}$, there exists $m$ such that $0=A X^{m}=\left[A,{ }_{m} N\right]$. But $N^{c} \subseteq A$ for some $c$, and hence $N^{c+m}=0$ whence $N=\psi(L) \subseteq \nu(L) \subseteq \psi(L)$ and the proof is complete.

For a Lie algebra $L$ and $b \in L$ we define

$$
E_{L}(b)=\{x \in L:[x, b]=0 \text { for some } n=n(x, b)\} \text {. }
$$

The Lie algebra $L$ is said to be an Engel algebra in case $L=E_{L}(b)$ for any $b$ in $L$.

The class Max is the class of all Lie algebras satisfying the maximal condition on subalgebras. By Amayo and Stewart [2], Max-cu $<$ Max. Furthermore by Amayo [1], $\mathbb{F} \cap \operatorname{Max}<\mathfrak{F} \cap \mathfrak{R}$, where $\mathbb{E}$ denotes the class of Engel Lie algebras.

Let $L \in \mathbb{S} \cap(\mathfrak{R} \mathrm{Max}-\mathrm{cu}) \cup \mathbb{S} \cap(\mathfrak{F} \mathrm{Max}-\mathrm{cu})$. Evidently $N=\nu(L) \in \mathfrak{N}$ and $L$ has good Frattini structure if and only if $L / N^{2}$ has good Frattini structure (see Amayo and Stewart [3, p. 133] and Proposition 2.1). Thus to show that $L$ has good Frattini structure we may assume that $\nu(L)$ is abelian.

Suppose if possible that $L$ does not have good Frattini structure. As $L$ satisfies the maximal condition for ideals (trivially if $L \in \mathbb{S} \cap$ (F Max-cu) and, by Amayo and Stewart [3, pp. 225-240], if $L \in \mathbb{S} \cap \mathfrak{N} M$ ax-cu and $\nu(L) \in \mathfrak{U}$ ), we may factor out an ideal $I$ of $L$ maximal with respect to $L / I$ not having good Frattini structure. Replacing $L$ by this quotient we may now assume that

(i) If $0 \neq J \triangleleft L$, then $L / J$ has good Frattini structure.

(ii) $A=\nu(L)$ is abelian. 
(iii) $L \in$ (S) and has an ideal $I$ such that $L / I \in$ Max-cu and either $I$ is abelian or else $I$ is finite dimensional.

Let $N=\psi(L), M=\tilde{\nu}(L)$ and $P=\rho(L)$. Then $A$ may or may not be zero. If $L \in \mathfrak{F}$ then by Barnes and Newell [4], $L$ has good Frattini structure, a contradiction. So $L$ is infinite dimensional.

Claim $1 . M<N$.

Let $B / C$ be a chief factor with $[B, M] \nsubseteq C$. If $C \neq 0$ then $L / C$ has good Frattini structure and $(M+C) / C \subseteq \tilde{\nu}(L / C)=\psi(L / C)$, hence $[B, M] \subseteq$ $C$. Suppose then that $C=0$. If $J \triangleleft L$ and $J \neq 0$, then $(B+J) / J$ is zero or a chief factor of $L / J$ and so $0 \neq[B, M] \subseteq B \cap J<B$. Thus

$$
B \subseteq \mu(L)=\cap\{J: 0 \neq J \triangleleft L\} .
$$

If $I=0$, then $L \in$ Max-cu, $B$ is an irreducible $L$-module and so $B$ is finite dimensional. If $I \neq 0$ and $I$ is finite dimensional, then $B \subseteq I$, so $B$ is finite dimensional. If $I \neq 0$ and $I$ is abelian, then $0 \neq B \subseteq I, I$ is a finitely generated $L / I$-module and, by Amayo and Stewart [3, pp. 225-240], this implies that $I$, and so $B$, is finite dimensional. So in all cases $B$ is finite dimensional and $L \in$ Max. Now let $x \in M$. Then for some $r$,

$$
[B, x]=\left[B,_{r+1} x\right]=\cdots=\bigcap_{i=1}^{\infty}[B, x]
$$

( $B$ is finite dimensional) and, since also $M \triangleleft L$ and $M^{c} \subseteq B$ for some $c$ $(L / B$ has good Frattini structure), we have

$$
\left[L,,_{c+1+r} x\right] \subseteq[B, r x]=\bigcap_{i=1}^{\infty}[B, i x] \subseteq\left[L,,_{c+2+r} x\right] .
$$

Thus with $s=c+r+1$, we have

$$
\left[L,,^{x}\right]=\left[L, s_{s+1} x\right] \text {, and, hence, } L=E_{L}(x)+[L, x]=E_{L}(x)+B \text {. }
$$

Let $D=C_{L}(B) \triangleleft L$. Then $L / D \in \mathfrak{F}$ and $L \notin \mathfrak{F}$, so $D \neq 0$ and $B \subseteq D$ (since $B \subseteq \mu(L))$. Then $D \neq B$, as otherwise, $L \in \mathfrak{F}$ and $D=D \cap\left(E_{L}(x)\right.$ $+B)=\left(D \cap E_{L}(x)\right)+B$. Evidently, $E_{L}(x) \cap D \triangleleft E_{L}(x)$ and $\left[E_{L}(x) \cap\right.$ $D, B]=0$, and so $0 \neq E_{L}(x) \cap D \triangleleft L$, so that $B \subseteq E_{L}(x) \cap D$. Therefore, $L=E_{L}(x)$ and $M=E_{M}(x)$, whence $M \in \mathbb{E} \cap \operatorname{Max}<\mathfrak{F} \cap \mathfrak{N}$. Thus $M=$ $\nu(L)<\psi(L)$, a contradiction which proves Claim 1 .

The proof of Claim 1 also shows that we may assume that:

(iv) $L$ has no minimal ideals (in particular, if $I \neq 0$ then $I$ is not finite dimensional).

(v) If $J_{1}, J_{2}$ are nonzero ideals of $L$, then $J_{1} \cap J_{2} \neq 0$.

(vi) $P=\rho(L)<\psi(L)$ (proved in the same way as Claim 1).

If now $I \neq 0$, then from above, $I$ is abelian and a finitely generated $L / I$ module under the action $u(x+I)=[u, x]$ for $u \in I$ and $x \in L$ and, 
evidently, $(N+I) / I \subseteq \psi(I ; U(L / I))$ and $L / I \in$ Max-cu and, hence, by Theorem 3.1, $[I, r, N]=0$ for some $r$. But $N^{c} \subseteq I$ for some $c(L / I$ has good Frattini structure) and, hence, $N^{c+r} \subseteq\left[I,{ }_{r} N\right]=0$, a contradiction. Similarly if $A=\nu(L) \neq 0$ we obtain a contradiction.

So we may finally assume that $I=0$ and $L \in \operatorname{Max}$-cu $\cap \Theta, \nu(L)=0$, $N=\psi(L) \supseteq \tilde{\nu}(L)+\rho(L)$. But then $L$ is finitely generated as a module over itself under the adjoint action and $N \subseteq \psi(L ; U(L))$, whence by Theorem 3.1, $0=L(N)^{r}=[L, r]$ for some $r$. Thus $N^{r+1}=0$ and $N<\nu(L)$, a contradiction.

So we have proved

THEOREM 4.1. If $L \in \mathbb{S} \cap(\mathfrak{R}$ Max-cu) or $L \in \mathbb{S} \cap(\mathfrak{F}$ Max-cu), then $L$ has good Frattini structure.

Since we do have $\mathfrak{F}<$ Max-cu in prime characteristic, Theorem 4.1 implies Theorem E. Further, Corollary F follows from Theorem D and the remarks in Stewart and Towers [8].

\section{REFERENCES}

1. R. K. Amayo, Engel Lie rings with chain conditions, Pacific J. Math. 54 (1974), 1-12.

2. R. K. Amayo and I. N. Stewart, Finitely generated Lie algebras, J. London Math. Soc. (2) 5 (1972), 697-703.

3. Infinite-dimensional Lie algebras, Noordhoff, Groningen, 1974.

4. D. W. Barnes and M. L. Newell, Some theorems on saturated homomorphs of soluble Lie algebras, Math. Z. 115 (1967), 231-234.

5. C. W. Curtis, Non-commutative extensions of Hilbert rings, Proc. Amer. Math. Soc. 4 (1953), 945-955.

6. N. J. Divinsky, Rings and radicals, Univ. of Toronto Press, 1965.

7. J. Dixmier, Algébres enoeloppantes, Cahier Scientifiques, fasc. 37, Gauthier-Villiars, Paris, 1974.

8. D. Towers and I. Stewart, The Frattini subalgebras of certain infinite-dimensional soluble Lie algebras, J. London Math. Soc. (2) 11 (1975), 207-215.

Department of Mathematics, University of Connecticutt, Storrs, CONnecticut 06268

Current address: Department of Mathematics, Southern Illinois University, Carbondale, Illinois 62901 